\title{
Sterile neutrinos in the $3+5$ scenario and solar data
}

\author{
João Pulido* \\ Centro de Física Teórica de Partículas, Instituto Superior Técnico, Av. Rovisco Pais, 1049-001, \\ Lisboa, Portugal \\ E-mail: pulido@cftp.ist.utl.pt

\section{C.R. Das} \\ Centro de Física Teórica de Partículas, Instituto Superior Técnico, Av. Rovisco Pais, 1049-001, \\ Lisboa, Portugal \\ and \\ Department of Physics, University of Jyväskylä, Survontie 9, Jyväskylä, Finland \\ E-mail: crdas@cftp.ist.utl.pt
}

The flatness of the SuperKamiokande neutrino electron scattering spectrum and the apparent downturn of the charged current spectrum in the SNO data which the Large Mixing Angle solution (LMA) to the solar neutrino problem fails to predict are analysed in the context of an extension to the standard electroweak model with light sterile neutrinos. It is found that a sterile neutrino which is quasi degenerate with the active ones with $\Delta m_{41}^{2}=10^{-5} \mathrm{eV}^{2}$ and mixing $\sin \theta_{14}=0.04$ provides a suitable improvement to the LMA data fits.

The European Physical Society Conference on High Energy Physics -EPS-HEP2013

18-24 July 2013

Stockholm, Sweden

\footnotetext{
* Speaker.
} 
The introduction of light sterile neutrinos into the Standard Model of electroweak interactions was motivated by the experimental observations from the LSND accelerator experiment to which the later data from other accelerator experiments like KARMEN, ICARUS, MiniBoone, along with Gallium calibration and reactor experiments provided additional evidence [1]. These are very short baseline experiments $(L \sim f e w \times 10 \mathrm{~m})$ for whose anomalous data an oscillation to one or two sterile neutrino states ( $\mathrm{s}=1$ or 2 ) seems to be implied with [2]

$$
\Delta m_{41}^{2}, \Delta m_{51}^{2}=O\left(\left|1 e V^{2}\right|\right) .
$$

Specifically, from accelerator experiments $\left(v_{e}\right.$ or $\bar{v}_{e}$ appearance from $v_{\mu}$ or $\left.\bar{v}_{\mu}\right)$ it is found that

$$
\sin ^{2} 2 \theta_{e \mu}=(4-10) \times 10^{-3}, \Delta m^{2}=(4-7) \times 10^{-1} e V^{2}
$$

while for the reactor and Gallium anomalies ( $v_{e}$ and $\bar{v}_{e}$ disappearance)

$$
\sin ^{2} 2 \theta_{e e}=(70-200) \times 10^{-3}, \Delta m^{2}=(2-3) \times 10^{-1} e V^{2}
$$

with the definitions $\sin ^{2} 2 \theta_{e \mu}=4\left|u_{e 4}\right|^{2}\left|u_{\mu 4}\right|^{2}, \sin ^{2} 2 \theta_{e e}=4\left|u_{e 4}\right|^{2}\left(1-\left|u_{e 4}\right|^{2}\right)$. Owing to such a large oscillation frequency, these sterile neutrinos do not play any role in solar neutrino oscillations.

It is usually argued on the other hand that the solar neutrino problem is 'solved' which is not the case. In fact, an estimation made by the Borexino Collaboration shows that there is a gap in the knowledge of the neutrino survival probability in the vacuum matter transition region $[O(1-5) \mathrm{MeV}]$ [3]. More importantly, besides the long standing problem of the flatness of the SuperKamiokande (SK) spectrum [4], [5] which the Large Mixing Angle (LMA) solution fails to explain, also the LMA charged current (CC) spectrum prediction from the SNO experiment [6] seems to proceed in the opposite direction from its LMA prediction.

Investigating a survival probability leading to an electron and $\mathrm{CC}$ spectra more consistent with the SK and SNO CC ones, we were lead to introduce light sterile neutrinos and search for possible ranges of $\Delta m_{\text {new }}^{2}$ and $\theta_{\text {new }}{ }^{1}$. Adequate probability profiles were found from oscillations to sterile neutrinos which are quasi degenerate with respect to the active ones $\left(\Delta m_{41}^{2}=10^{-5} \mathrm{eV}^{2}\right)$ and with small mixing to these. Hence they are different from the sterile neutrinos that are suggested by accelerator, reactor and Gallium anomalies. Our $4 \times 4$ Hamiltonian describing the solar neutrino oscillations is in the weak basis

$$
\left(H_{I}\right)_{W}=U\left(\begin{array}{cccc}
0 & 0 & 0 & 0 \\
0 & \frac{\Delta m_{21}^{2}}{2 E} & 0 & 0 \\
0 & 0 & \frac{\Delta m_{31}^{2}}{2 E} & 0 \\
0 & 0 & 0 & \frac{\Delta m_{41}^{2}}{2 E}
\end{array}\right) U^{\dagger}+\left(\begin{array}{cccc}
V_{C C} & 0 & 0 & 0 \\
0 & 0 & 0 & 0 \\
0 & 0 & 0 & 0 \\
0 & 0 & 0 & -V_{N C}
\end{array}\right)
$$

where $U$ is the straightforward $4 \times 4$ extension of the usual leptonic mixing matrix, $V_{C C}=G_{F} \sqrt{2} N_{e}$, $V_{N C}=-G_{F} / \sqrt{2} N_{n}$ with $N_{e}, N_{n}$ denoting the electron and neutron densities. We use the representation $U=U_{34} \tilde{U}_{24} \tilde{U}_{14} U_{23} \tilde{U}_{13} U_{12}$. At this early stage of sterile neutrino investigation for the solar case we assume all sterile mixings to be equal with $\sin \theta_{41}=0.04$. We thus get the model survival probability shown in fig. 1 where also the LMA probability is displayed for comparison. As for the

\footnotetext{
${ }^{1}$ A similar investigation was performed by the authors of refs.[8],[9].
} 


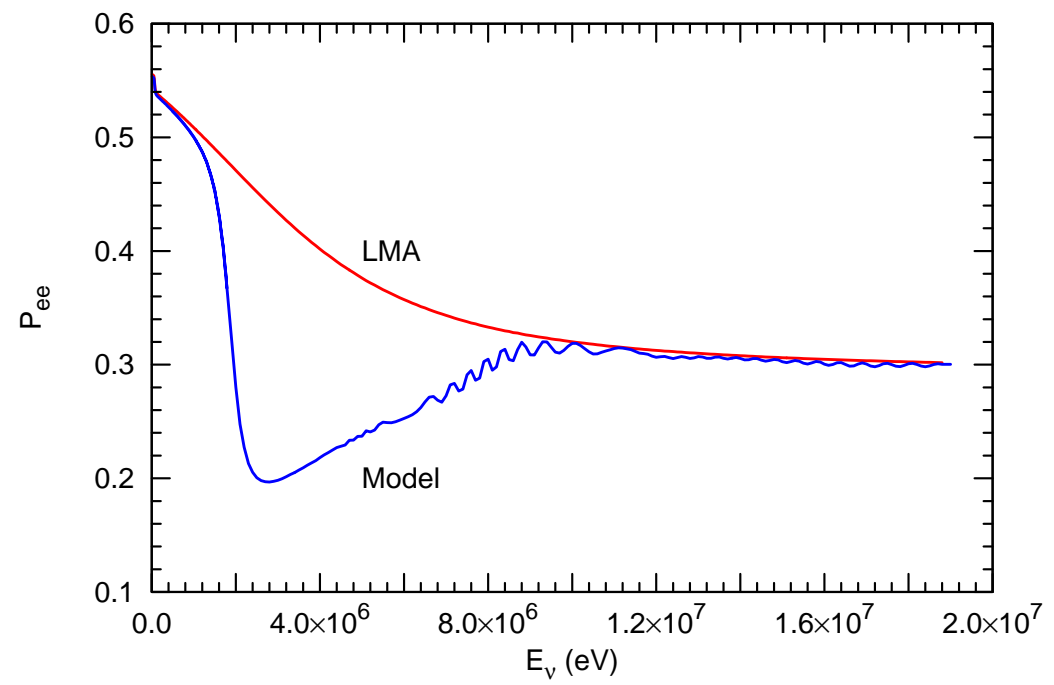

Figure 1: Electron neutrino survival probability: LMA (upper curve) and sterile model (lower curve).

relevant physical quantities, we start with the SNO CC spectrum evaluated as

$$
R_{C C}\left(T_{e f f}\right)=\frac{\int_{Q}^{E_{\max }} \frac{d \phi_{v}(E)}{d E} P(E) \int_{m_{e}}^{E-\left(Q-m_{e}\right)} R\left(T_{e f f}, T\right) \frac{d \sigma_{C C}}{d T_{e f f}} d T d E}{P(E) \rightarrow 1}
$$

(see fig.2) where $Q=1.442 \mathrm{MeV}$ and $T, T_{\text {eff }}$ are the physical and measured kinetic energy of the electron. In eq.(5) $R\left(T_{e f f}, T\right)$ is the energy resolution function and the rest of the notation is standard. For the solar neutrino fluxes we used the AGSS09ph model [7]. We have also evaluated

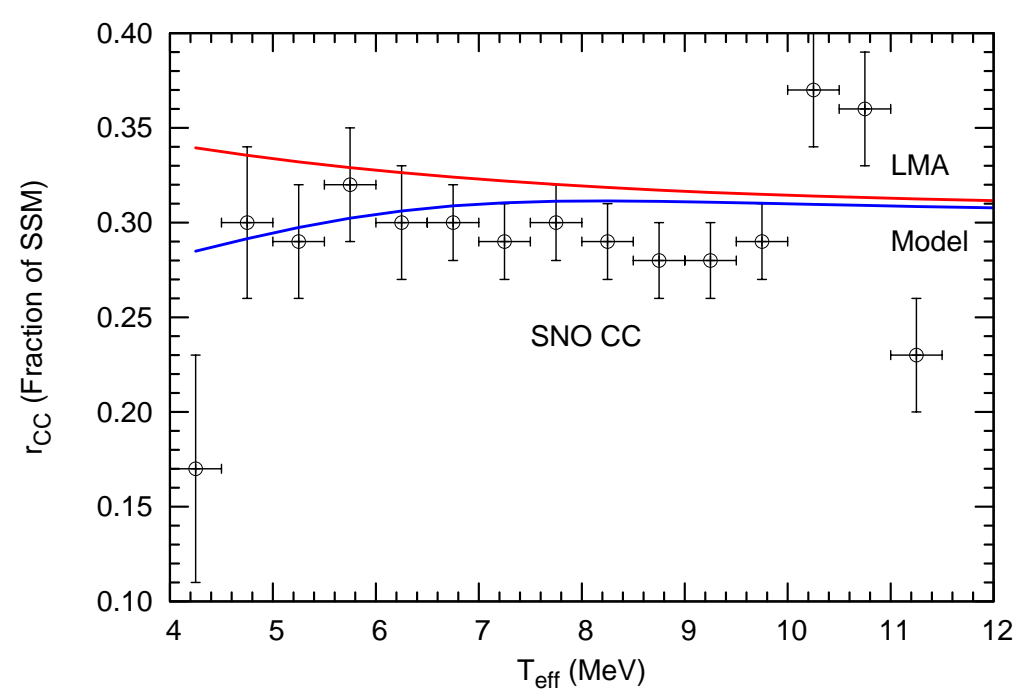

Figure 2: CC spectrum in SNO: the LMA prediction (upper curve), the model prediction (lower curve) and the data [6]. 
the electron scattering spectrum (ES) for SNO, SK and Borexino from the expression

$$
R_{E S}\left(E_{e f f}\right)=\frac{\int_{m_{e}}^{E_{e m a x}} d E_{e} R\left(E_{e f f}, E_{e}\right) \int_{E_{m}}^{E_{M}} d E \phi_{v}(E)\left[P_{e e}(E) \frac{d \sigma_{e}}{d E_{e}}+\left(P_{e \mu}(E)+P_{e \tau}(E)\right) \frac{d \sigma_{\mu, \tau}}{d E_{e}}\right]}{\int_{m_{e}}^{E_{e m a x}} d E_{e} R\left(E_{e f f}, E_{e}\right) \int_{E_{m}}^{E_{M}} d E \phi_{v}(E) \frac{d \sigma_{e}}{d E_{e}}}
$$

where $E, E_{e f f}$ are the physical and measured electron energy. Its LMA and model predictions are shown together with the SK data from 2010 (fig.3) and 2008 (fig.4). The downturn in the CC data for the lower energies is clearly seen in fig. 2 and a hint of the same effect can also be seen in the ES scattering data, especially in the second set (fig.4). Such an effect which the LMA model alone fails to account for, is clearly predicted by the sterile model.

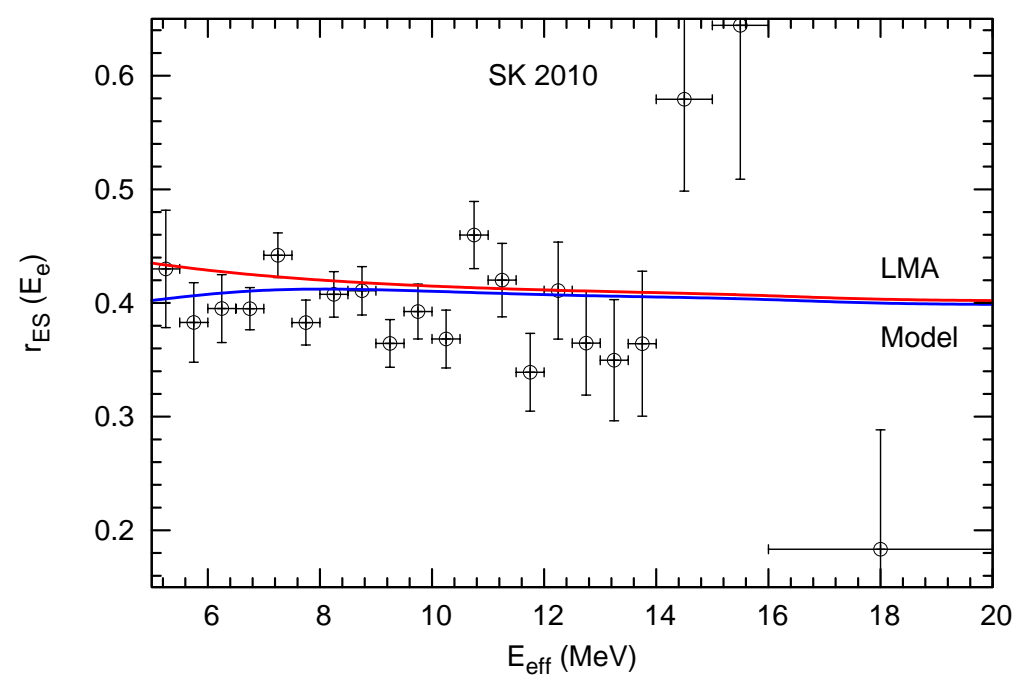

Figure 3: ES scattering in SK: the 2010 data [4]. The upper and lower curves are the LMA and sterile model predictions respectively.

We also evaluated the model predictions for the total rates which are given in table I along with the LMA ones and the data.

\begin{tabular}{|c|c|c|c|c|c|c|c|}
\hline & $\begin{array}{c}G a(A l l, \leq \\
D e c .2007) \\
\quad(S N U)\end{array}$ & $\begin{array}{c}C l \\
(S N U)\end{array}$ & $\begin{array}{c}S N O(C C) \\
\quad\left(\times 10^{6}\right. \\
\left.\mathrm{cm}^{2} s^{-1}\right)\end{array}$ & $\begin{array}{c}S N O(N C) \\
\left(\times 10^{6}\right. \\
\left.\mathrm{cm}^{2} s^{-1}\right)\end{array}$ & $\begin{array}{c}S N O(E S) \\
\quad\left(\times 10^{6}\right. \\
\left.\mathrm{cm}^{2} \mathrm{~s}^{-1}\right)\end{array}$ & $\begin{array}{c}S K \\
\left(\times 10^{6}\right. \\
\left.\mathrm{cm}^{2} \mathrm{~s}^{-1}\right)\end{array}$ & $\begin{array}{c}\text { Borexino } \\
\left(\times 10^{6}\right. \\
\left.\mathrm{cm}^{2} \mathrm{~s}^{-1}\right)\end{array}$ \\
\hline \multirow{3}{*}{ Data } & 66.1 & 2.56 & 1.67 & 5.54 & 1.77 & 2.32 & 2.40 \\
\hline & \pm 3.1 & \pm 0.16 & $\pm_{0.04}^{0.05}$ & $\pm_{0.31}^{0.33}$ & $\pm_{0.21}^{0.24}$ & \pm 0.04 & \pm 0.4 \\
\hline & & \pm 0.15 & $\pm_{0.08}^{0.07}$ & $\pm_{0.34}^{0.36}$ & $\begin{array}{l} \pm_{0.10}^{0.09} \\
\end{array}$ & \pm 0.05 & \pm 0.1 \\
\hline$L M A$ & 62.4 & 2.70 & 1.69 & 5.22 & 2.21 & 2.21 & 2.27 \\
\hline Model & 61.0 & 2.60 & 1.61 & 5.13 & 2.14 & 2.14 & 2.12 \\
\hline
\end{tabular}

Table I: the data and the LMA and model predictions for the total rates. Units are in SNU for Gallium and Chlorine and in $10^{6} \mathrm{~cm}^{2} s^{-1}$ for the remainder. For Ga all data from SAGE and Gallex/GNO are included up to Dec.2007 
We next perform an analysis of the quality of the fits to the data. Using the standard $\chi^{2}$ definition [10]

$$
\chi^{2}=\sum_{j_{1}, j_{2}}\left(R_{j_{1}}^{t h}-R_{j_{1}}{ }^{\exp }\right)\left[\sigma^{2}(t o t)\right]_{j_{1} j_{2}}^{-1}\left(R_{j_{2}}^{t h}-R_{j_{2}}{ }^{\exp }\right)
$$

where indices $j_{1}, j_{2}$ run over the 7 solar neutrino experiments and the error matrix includes the cross section, the astrophysical and the experimental uncertainties, we obtain for the rates only, with $\Delta m_{\text {sterile }}^{2}$ and $\theta_{\text {sterile }}$ as free parameters,

$$
\chi_{\text {rates }}^{2}(\mathrm{LMA})=8.1 / 5 \text { d.o.f. } \quad, \quad \chi_{\text {rates }}^{2}(\operatorname{model})=15.3 / 5 \text { d.o.f. }
$$

A word of caution must be inserted here as regards the inclusion of the Ga rate, since its contribution to $\chi_{\text {rates }}^{2}$ is overwhelming. Had we taken for instance the Ga/GNO data only from the period 1998$2003(62.9 \pm 5.4 \pm 2.5 \mathrm{SNU})$ the result would be

$$
\chi_{\text {rates }}^{2}(\mathrm{LMA})=5.5 / 5 \text { d.o.f. } \quad, \quad \chi_{\text {rates }}^{2}(\text { model })=12.5 / 5 \text { d.o.f }
$$

\begin{tabular}{|c|c|c|}
\hline $\begin{array}{l}\text { GallexI } \\
\text { GallexII } \\
\text { GallexIII }\end{array}$ & $\begin{array}{c}\leq \text { June } 1992 \\
\text { Aug }^{\prime} 92 \rightarrow \text { Jun }^{\prime} 94 \\
\text { Oct }^{\prime} 94 \rightarrow \text { Oct }^{\prime} 95\end{array}$ & \begin{tabular}{|l}
$83 \pm 19$ \\
$46 \pm 10$ \\
$54 \pm 11$
\end{tabular} \\
\hline & $1991-97$ & $1998-03$ \\
\hline $\begin{array}{c}\text { Gallex/GNO } \\
\text { SAGE }\end{array}$ & $\begin{array}{l}77.5 \pm 6.2 \pm_{4.7}^{4.3} 62 . \\
79.2 \pm 8.6 \pm_{4.7}^{4.3}\end{array}$ & $\begin{array}{l}2.9 \pm 5.4 \pm 2.5 \\
63.9 \pm 5.0\end{array}$ \\
\hline 2003 & 2004 & 2006 \\
\hline
\end{tabular}

so $\chi_{\text {rates }}^{2}$ strongly depends on the Ga data period one considers. Moreover the Ga rate has been decreasing all along its history of data taking (see table II), a fact whose origin remains unclear.

Table II: the evolution of the Ga rate over time (units are in SNU).

Hence the above $\chi_{\text {rates }}^{2}$ values may well be meaninglessly high and misleading. Removing the Ga rate from the calculation, one gets instead

$$
\chi_{\text {rates }}^{2}(\mathrm{LMA})=5.7 / 4 \text { d.o.f. } \quad, \quad \chi_{\text {rates }}^{2}(\text { model })=5.9 / 4 \text { d.o.f } \quad[\text { no Ga rate }]
$$

and thus fits of equivalent quality for LMA alone and the sterile neutrino models. Turning now to the spectral fits, we have for the SNO CC spectrum (see fig.2)

$$
\chi_{\mathrm{CC} \text { spectrum }}^{2}(\mathrm{LMA})=24.0 / 13 \text { d.o.f. } \quad, \quad \chi_{\mathrm{CC} \text { spectrum }}^{2}(\text { model })=21.6 / 13 \text { d.o.f }
$$

where we took into account all 15 data points. As expected, the sterile neutrino model fits the data better than LMA, as it reflects the downturn of the spectrum for the lower energies. Regarding the ES spectrum, it should be noted that not only this appears to be flat, but there is also a hint for a 


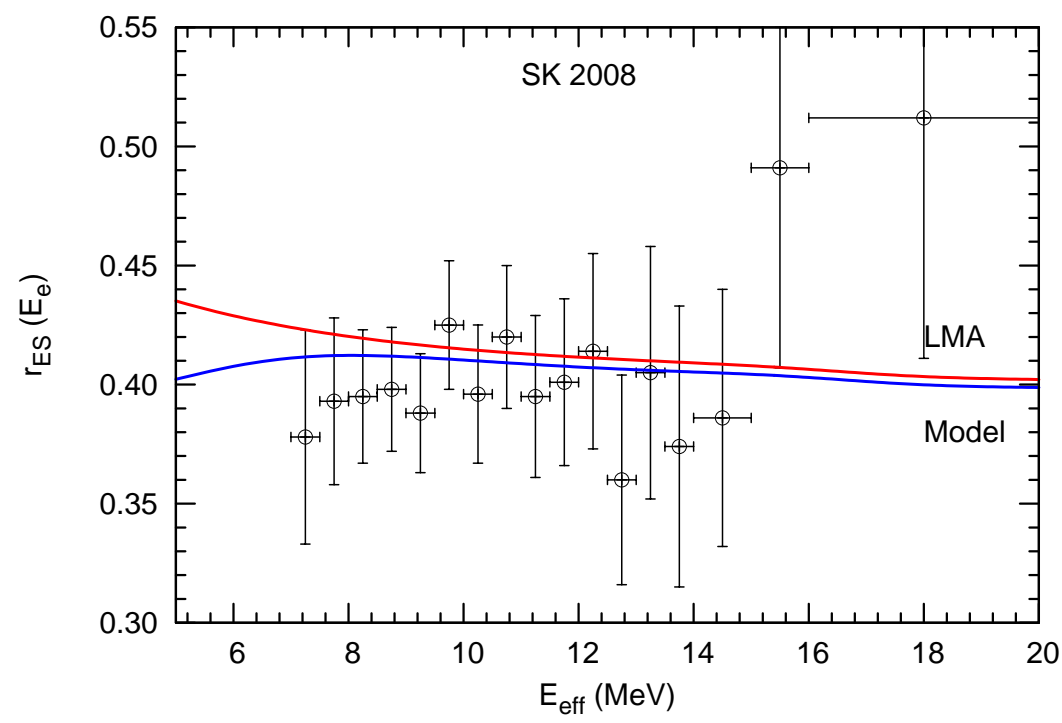

Figure 4: The same as fig. 3 for the 2008 data [5].

downturn at the lower energies (see figs.3 and 4). To this end we performed as well a $\chi^{2}$ analysis for both SK data sets. For the 2010 data (fig.3) we find ${ }^{2}$

$$
\chi_{\mathrm{ES} \text { spectrum }}^{2}(\mathrm{LMA})=19.2 / 16 \text { d.o.f. } \quad, \quad \chi_{\mathrm{ES} \text { spectrum }}^{2}(\operatorname{model})=19.5 / 16 \text { d.o.f }
$$

so that the two fits look similar for this set. For the 2008 data (fig.4) on the other hand

$$
\chi_{\mathrm{ES} \text { spectrum }}^{2}(\mathrm{LMA})=3.6 / 12 \text { d.o.f. } \quad, \quad \chi_{\mathrm{ES} \text { spectrum }}^{2}(\text { model })=2.6 / 12 \text { d.o.f }
$$

and thus a better fit for the sterile model, as expected. Note that the smaller magnitude of the $\chi^{2}$ 's in the 2008 data is a consequence of both the smaller number of degrees of freedom and the fact that the predictions, especially the sterile model one, lie practically all within the data error bars, which is by no means the case for the 2010 data set.

We next summarize our conclusions:

- We still need to fill the gap in our knowledge of the solar neutrino survival probability in the intermediate energy range, the vacuum matter transition region.

- The LMA prediction seems to point in the wrong direction at the low energy end of the electron spectra, especially the charged current one.

- Oscillations to a sterile neutrino which is almost degenerate with the active ones with $\Delta m_{41}^{2}=$ $10^{-5} \mathrm{eV}^{2}$ and $\sin \theta_{14}=0.04$ seem to provide a solution to these inconsistencies.

- Inserting such a sterile neutrino, which would be the 5th or 6th neutrino, adds to the already confusing situation concerning sterile neutrino scenarios.

\footnotetext{
${ }^{2}$ In eqs.(12) and (13) we have not taken into account the highest energy data points in the calculation in view of their poorer statistics.
} 
- However from the experience with neutrino oscillations, we have learned that a confusing picture at the start may eventually emerge, after an accumulation of experimental tests for several years, as a clear and positive one. This may well be the case with the sterile neutrino extensions of the electroweak standard model.

\section{Acknowledgments}

C.R. Das acknowledges a scholarship from the Fundação para a Ciência e a Tecnologia (FCT, Portugal) (ref. SFRH/BPD/41091/2007), also greatly thanks the Department of Physics, Jyväskylä University, in particular Prof. Jukka Maalampi (HOD) for hospitality and financial support. This work was partially supported by FCT through the projects CERN/FP/123580/2011, PTDC/FISNUC/0548/2012 and CFTP-FCT Unit 777 (PEst-OE/FIS/UI0777/2013) which are partially funded through POCTI (FEDER).

\section{References}

[1] For a review on sterile neutrinos see: K. N. Abazajian, M. A. Acero, S. K. Agarwalla, A. A. Aguilar-Arevalo, C. H. Albright, S. Antusch, C. A. Arguelles and A. B. Balantekin et al., arXiv:1204.5379 [hep-ph].

[2] J. Kopp, P. A. N. Machado, M. Maltoni and T. Schwetz, JHEP 1305 (2013) 050 [arXiv:1303.3011 [hep-ph]].

[3] G. Bellini et al. [Borexino Collaboration], Phys. Rev. Lett. 108 (2012) 051302 [arXiv:1110.3230 [hep-ex]].

[4] K. Abe et al. [Super-Kamiokande Collaboration], Phys. Rev. D 83 (2011) 052010 [arXiv:1010.0118 [hep-ex]].

[5] J. P. Cravens et al. [Super-Kamiokande Collaboration], Phys. Rev. D 78 (2008) 032002 [arXiv:0803.4312 [hep-ex]].

[6] B. Aharmim et al. [SNO Collaboration], Phys. Rev. C 81 (2010) 055504 [arXiv:0910.2984 [nucl-ex]].

[7] A. M. Serenelli, Astrophys. Space Sci. 328 (2010) 13 [arXiv:0910.3690 [astro-ph.SR]].

[8] P. C. de Holanda and A. Y. .Smirnov, Phys. Rev. D 69 (2004) 113002 [hep-ph/0307266].

[9] P. C. de Holanda and A. Y. .Smirnov, Phys. Rev. D 83 (2011) 113011 [arXiv:1012.5627 [hep-ph]].

[10] C. R. Das, J. Pulido and M. Picariello, Phys. Rev. D 79 (2009) 073010 [arXiv:0902.1310 [hep-ph]]. 